\title{
MODULUS OF CONTINUITY OF THE QUANTUM $f$-ENTROPY WITH RESPECT TO THE TRACE DISTANCE
}

\author{
IOSIF PINELIS
}

Abstract. A well-known result due to Fannes is a certain upper bound on the modulus of continuity of the von Neumann entropy with respect to the trace distance between density matrices; this distance is the maximum probability of distinguishing between the corresponding quantum states. Much more recently, Audenaert obtained an exact expression of this modulus of continuity.

In the present note, Audenaert's result is extended to a broad class of entropy functions indexed by arbitrary continuous convex functions $f$ in place of the Shannon-von Neumann function $x \mapsto x \log _{2} x$. The proof is based on the Schur majorization.

Mathematics subject classification (2020): Primary 15A42, 26B25, 26D15, 47A30, 47A63, 47B06, 94A17; Secondary 47A60, 47B15, 47B65, 47A70.

Keywords and phrases: Modulus of continuity, density matrices, trace distance, von Neumann entropy, quantum $f$-entropy, convex functions, Schur majorization.

\section{REFERENCES}

[1] S. ARImoto, Information-theoretical considerations on estimation problems, Information and Control, 19: 181-194, 1971.

[2] K. M. R. AudenaERT, A sharp continuity estimate for the von Neumann entropy, Journal of Physics A: Mathematical and Theoretical, 40 (28): 8127-8136, Jun 2007.

[3] R. BhatiA, Matrix analysis, volume 169 of Graduate Texts in Mathematics, Springer-Verlag, New York, 1997.

[4] P. M. BlaU AND J. E. SCHWARTZ, Crosscutting social circles: testing a macrostructural theory of intergroup relations, Routledge, London, 1st edition, 2018., Description based on CIP data; resource not viewed.

[5] M. FAnNES, A continuity property of the entropy density for spin lattice systems, Comm. Math. Phys., 31 (4): 291-294, 1973.

[6] M. Gell-Mann And C. Tsallis, editors, Nonextensive entropy - interdisciplinary applications, Santa Fe Institute Studies in the Sciences of Complexity, Oxford University Press, New York, 2004.

[7] A. Gleason, Measures on the closed subspaces of a Hilbert space, Indiana Univ. Math. J., 6: 885$893,1957$.

[8] E. P. HAnson And N. DatTA, Tight uniform continuity bound for a family of entropies, 2017. arXiv:1707.04249 [quant-ph].

[9] E. P. HANSON AND N. DATTA, Maximum and minimum entropy states yielding local continuity bound, J. Math. Phys., 59 (4): 042204, 31, 2018.

[10] E. P. HANSON AND N. DATTA, Universal proofs of entropic continuity bounds via majorization flow, 2019, arXiv:1909.06981 [quant-ph].

[11] L. Jos T, Entropy and diversity, Oikos, 113 (2): 363-375, 2006.

[12] A. W. Marshall, I. Olkin, And B. C. ARnOld, Inequalities: theory of majorization and its applications, Springer Series in Statistics. Springer, New York, second edition, 2009.

[13] M. A. Nielsen AND I. L. ChUANG, Quantum computation and quantum information, Cambridge University Press, Cambridge, 2000. 
[14] I. PInELIS, Generalized semimodularity: Order statistics, In High dimensional probability VIII, volume 74 of Progress in Probability, pages 99-119, Springer, 2019.

[15] I. PINELIS, Modulus of continuity of the quantum $f$-entropy with respect to the trace distance, 2021. arXiv:2107.10112 [quant-ph].

[16] V. Sbordoni, G. Allegrucci, And D. Ces Aroni, Population structure, In W. B. White and D. C. Culver, editors, Encyclopedia of Caves (Second Edition), pages 608-618, Academic Press, Amsterdam, second edition, 2012.

[17] C. Ts allis, Possible generalization of Boltzmann-Gibbs statistics, Journal of Statistical Physics, 52 (1): 479-487, Jul 1988.

[18] C. TSALLIS, Introduction to nonextensive statistical mechanics: approaching a complex world, Springer, New York, NY, 2010.

[19] Z. ZHANG, Uniform estimates on the Tsallis entropies, Lett. Math. Phys., 80 (2): 171-181, 2007. 